
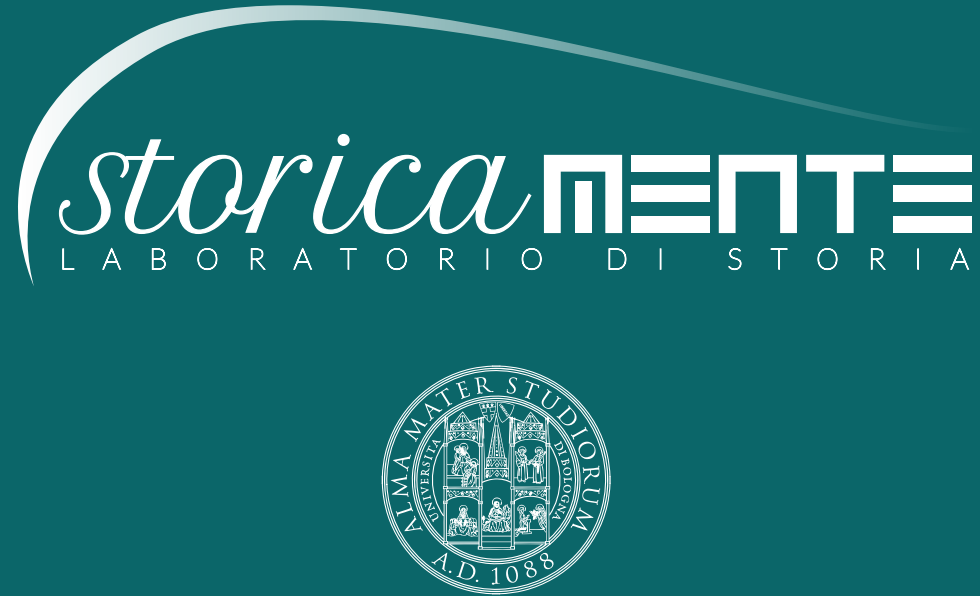

ALMA MATER STUDIORUM

Università di Bologna

Dipartimento di Storia Culture Civiltà

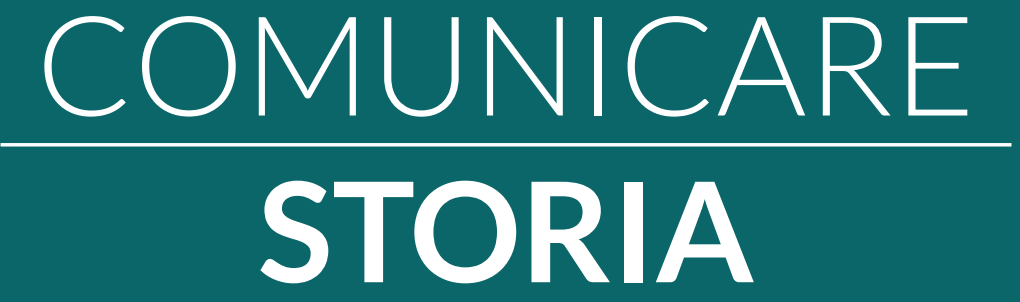




\section{STORICAMENTE.ORG}

\section{Laboratorio di Storia}

Giancarlo Poidomani

Serie Tv e Public History: l'immaginario storico americano in The Walking Dead

Numero 14 - 2018

ISSN: 1825-411X

Art. 8

pp. 1-24

DOI: $10.12977 /$ stor698

Editore: BraDypUS

Data di pubblicazione: 16/03/2018

Sezione: Comunicare storia 


\title{
Serie Tv e Public History: l'immaginario storico americano in The Walking Dead
}

\author{
GIANCARLO POIDOMANI
}

Univ. degli Studi di Catania, Dipartimento di Scienze

Politiche e Sociali

The article analyzes the American historical imaginary present in The Walking Dead. In this tv-serie, history is a subtext in which individual characters embody some key-moments in American and, in general, in Western Europe history: the pioneers, the myth of the frontier, the conquest of the West, the genocide of the Native Americans, the difficult construction of democracy in the nineteenth century, the contrast between nature and industrial civilization, the Civil War and soon.

\section{Premessa}

Nel giugno del 2017 è stata costituita a Ravenna la prima Associazione Italiana di Public History (AIPH). Essa è nata dall'esigenza di "fare storia", coniugando i percorsi di ricerca "con un'avveduta metodologia scientifica $[\ldots]$ attraverso i linguaggi molteplici della storia rinvenibili nell'odierna società della comunicazione» [Ridolf 2017, 9]. Per costruire una storia che "appartenga" al pubblico, gli storici devono rinnovare le forme di narrazione delle loro ricerche in modo tale da raggiungere audiences esterne all'ambito accademico e misurandosi con tutto ciò che 
passa nei mass media, soprattutto quelli di più larga diffusione come la tv e internet [Garofalo, Roghi 2015, 11-15].

Uno degli obiettivi del public historian è quello di interpretare e contestualizzare memorie collettive che fanno parte di «un passato che è attivo nel nostro presente» [Noiret 2017, 17], facendo una «storia in contatto diretto con l'evoluzione della mentalità e del senso delle appartenenze collettive delle diverse comunità che convivono all'interno dello spazio nazionale e nel villaggio globale e valorizzare lo studio delle loro identità» [Noiret 2009, 275].

Se dunque la Public History si basa su una serie di pratiche capaci di rendere più consapevole il rapporto di una società con il passato», illuminando e rendendo "più e meglio visibile non solo la cosa di volta in volta guardata, ma anche la logica, culturale e discorsiva, dei diversi sguardi di volta in volta implicati e depositati sulla cosa, come un sedimento» [Cipolloni 2017, 243], allora rintracciare gli elementi di un immaginario storico collettivo in alcune serie tv mainstream potrebbe rappresentare uno dei tanti strumenti a disposizione di un public historian.

\section{The Walking Dead e l'immaginario storico americano}

T-Dog: «Credo che il mondo sia cambiato».

Guillermo: «No, è sempre lo stesso di prima. Il debole viene schiacciato».

Vatos, I/4

Giunta alla sua ottava stagione, la serie tv nata dal graphic novel di Robert Kirkmann, curata e sceneggiata dal suo stesso autore insieme a Frank Darabont, Glen Mazzara e Scott M. Gimple, conta oggi milioni di spettatori e di fan.

Il protagonista, il vicesceriffo della contea di King Rick Grimes, si risveglia in ospedale, dopo essere stato ferito in un conflitto a fuoco con 


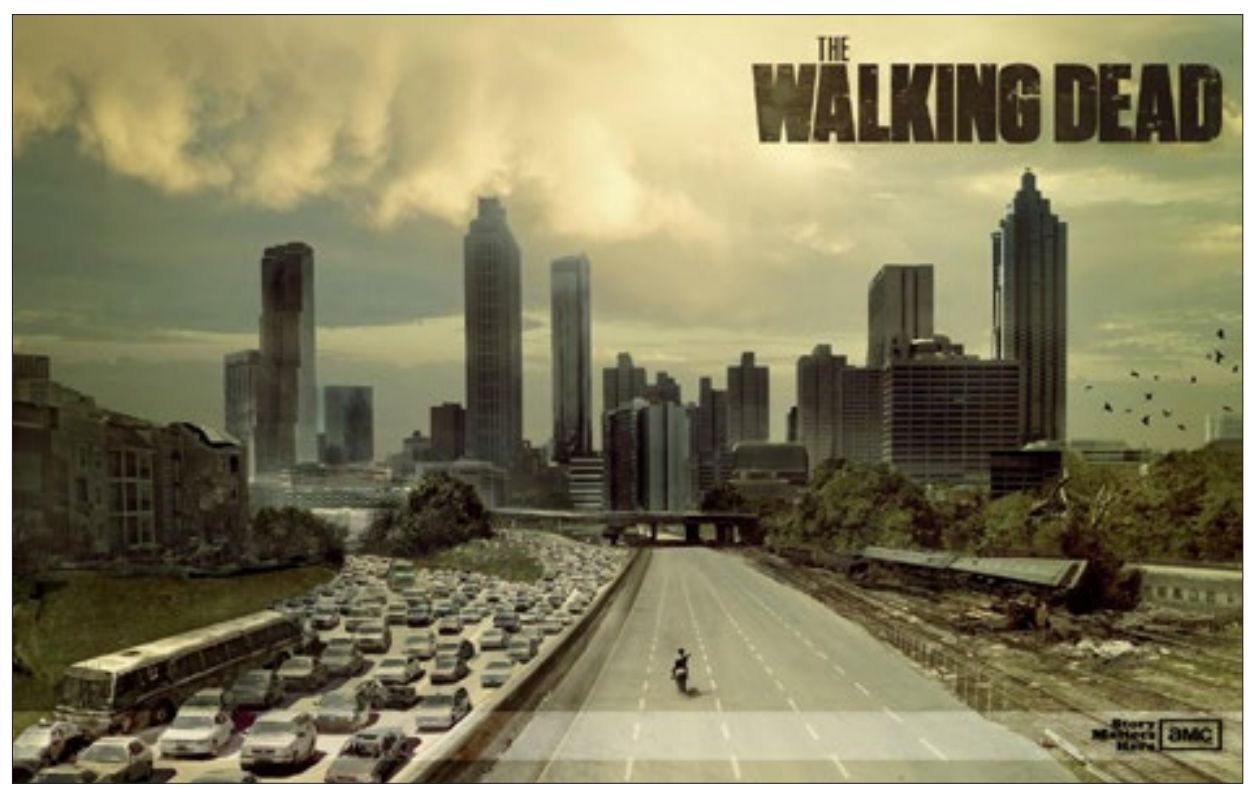

Manifesto della serie-TV The-Walking-Dead

alcuni rapinatori, e scopre che il mondo al quale era abituato non esiste più. Gli zombi hanno invaso l'America (forse tutta la terra).

La storia è la vicenda di un gruppo di uomini e donne, sopravvissuti a una "epidemia zombi”, costretti a confrontarsi con se stessi, con i propri limiti e paure, in un mondo ostile, infestato da zombi affamati di carne umana e popolato da altri sopravvissuti.

I protagonisti di TWD devono riscrivere le regole del vivere civile in un mondo nel quale bisogna ritornare ai bisogni essenziali. Essi sono costretti a ricostruire una morale in un mondo completamente stravolto rispetto a quello precedente, caratterizzato da penuria di risorse, assenza di rifugi sicuri, scomparsa di qualsiasi tipo di legge e di autorità. Ogni giorno devono confrontarsi con la propria coscienza e devono “evolversi" per non soccombere. Quello di TWD è un mondo nuovo, con le sue leggi e le sue regole ma, allo stesso tempo, ci dice molte cose di quello attuale. Guardando oltre la superficie dell'horror, del sangue e dello splatter, è possibile individuare in TWD riferimenti a importanti 
tematiche: religiose, scientifiche, etiche [Poli 2015, 22-24], storiche, politiche e culturali.

Al centro della narrazione di TWD non è soltanto l'umanità di una futura, potenziale, paventata apocalisse ma l'uomo contemporaneo con i suoi problemi, i suoi dilemmi, il suo rapporto con la natura, la scienza, la fede, l'altro e il "diverso".

Gli zombi incarnano la paura del diverso, dello straniero in tempi nei quali la porosità delle frontiere (rese ancora più labili dal progresso dei trasporti e delle comunicazioni) si somma a chiusure nazionalistiche ed egoistiche. Da questo punto di vista, TWD sembra calzare a pennello come rappresentazione di quella invasione dell'immaginario collettivo da parte di un'America che lo fa, ha scritto Dominique Moïsi attraverso la denuncia dei suoi punti deboli, proprio «nel momento in cui (l'America n.d.r.) non ha più la volontà o i mezzi per essere il gendarme del mondo» [Moïsi 2016]. È la cosiddetta "geopolitica delle serie" che, secondo Moïsi, invece di promuovere i valori di libera circolazione delle idee, delle persone e delle merci, aperta e solidale, diffonde insicurezza, paura, diffidenza verso l'altro.

È indubbio che il ritorno degli zombi, dopo il grande successo degli anni '60-'70, sia stato rafforzato dal clima di insicurezza e di pericolo seguente l'attacco alle Twin Towers dell'11 settembre 2001.

Il successo della serie tv, a partire dal 2010, riflette inoltre le incertezze della grave crisi economica iniziata nel 2007-2008, che ha sgretolato certezze e valori, facendo perdere agli uomini ogni sicurezza economica.

A essere messa in discussione, negli ultimi decenni, è stata proprio quella stessa tecnologia che tanti progressi ha determinato rendendo spesso più confortevole e più sicura la vita degli uomini. Ed è stata proprio una distorsione e una aberrazione tecnologica a provocare l'epidemia zombi. Una concretizzazione di tutti i timori provocati dalle "emergenze sanitarie" che si sono susseguite negli ultimi decenni: l'Aids; la "sindrome della mucca pazza", la SARS, l'influenza aviaria, la febbre suina e quelle più recenti (Ebola, ecc). Emergenze nelle quali, nella 
maggior parte dei casi, i mass media hanno dimostrato la tendenza ad allarmare la popolazione e a cavalcare il sensazionalismo piuttosto che a informare con obiettività.

\section{II mondo post-apocalittico e il passato}

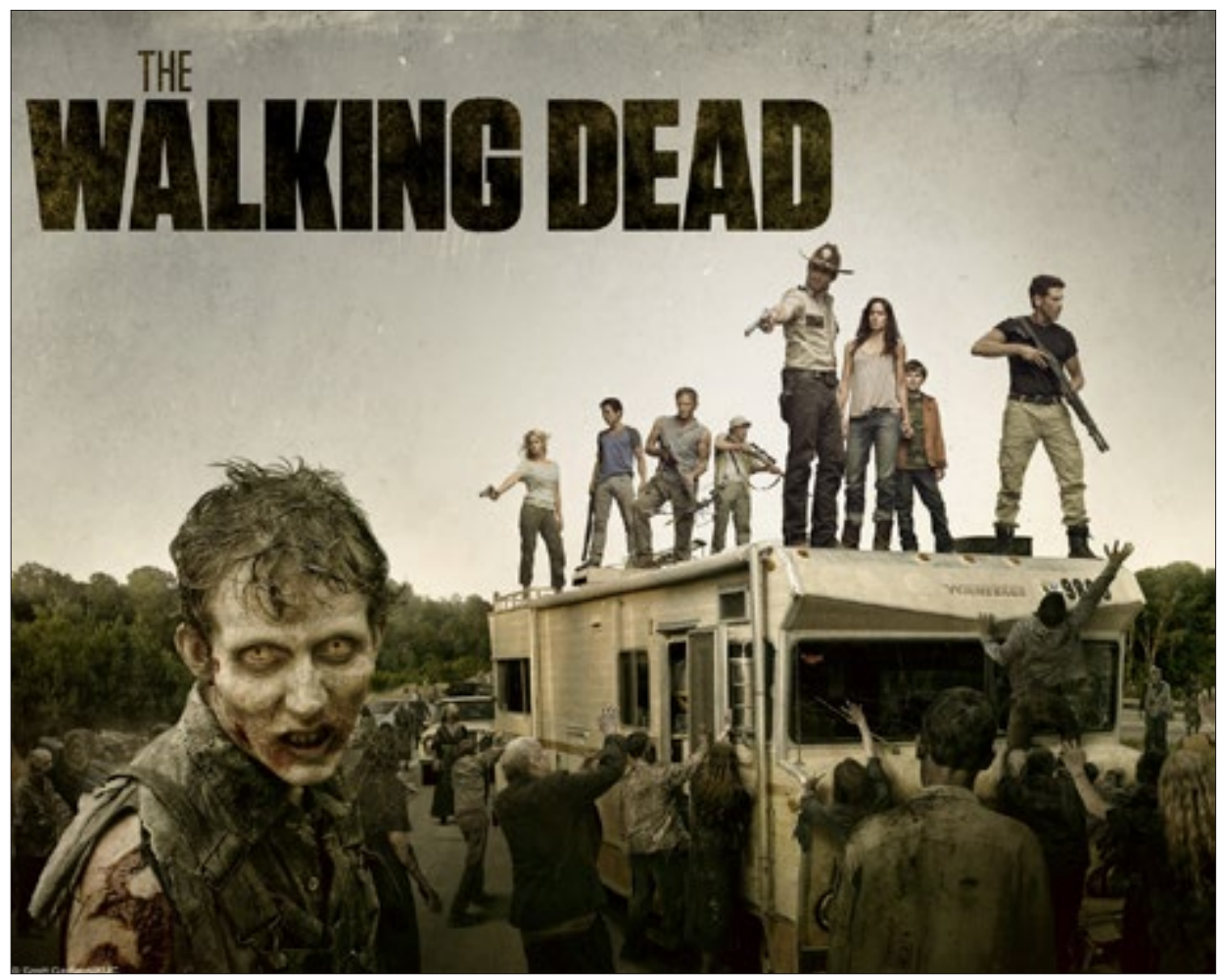

Il mondo apolcalittico in The Walking Dead

Dunque un mondo post-apocalittico, quello di TWD.

$\mathrm{E}$, di fronte a un mondo che non è più lo stesso di prima e a un futuro incerto, l'uomo è costretto a guardare al passato. A quale passato guardano i sopravvissuti di TWD?

La serie si richiama ad alcuni capisaldi narrativi ben presenti nell'immaginario storico americano, con riferimenti ai principali eventi, pro- 
cessi, personaggi del processo di costruzione dello Stato e della nazione americana.

Nel 1994 questo “canone" storiografico venne sistematizzato nei National Standards for United States History, una raccolta di problemi, idee e concetti relativi alla storia degli Stati Uniti che ogni studente americano tra i 10 e i 18 anni avrebbe dovuto conoscere. Il progetto era nato sotto le amministrazioni repubblicane di Reagan e Bush ed era stato portato a termine durante la presidenza Clinton. Ma l'approccio storico-politico che vi stava alla base era più vicino a quello della cultura liberal e progressista. Nei National Standards la storia veniva considerata come una disciplina di formazione civica, una risorsa strategica in una società democratica, precondizione di una corretta informazione politica [Testi 1996, 7-53].

In TWD troviamo citati, spesso in modo quasi subliminale, i più importanti eventi, personaggi e processi della periodizzazione proposta dai National Standards: i pionieri e il processo fondativo dello Stato e della nazione americana; l'epopea della frontiera e della conquista del West; il genocidio dei nativi americani; la guerra civile e la segregazione razziale; la nascita della superpotenza americana nel XX secolo; il confronto tra la democrazia e i totalitarismi novecenteschi; la controcultura; la tensione verso la wilderness e la cultura nonviolenta; il femminismo.

Vi troviamo trattati poi concetti e tematiche tipiche della storia contemporanea: statualità, leadership e potere, massa e individuo, uso e abuso delle armi, pena di morte, scontro tra democrazia e totalitarismi, competizione per le risorse, rapporto tra scienza e potere e tra scienza e fede. I riferimenti a molti dei momenti di costruzione dello Stato e della nazione americana sono particolarmente interessanti in una serie che mette in scena proprio il collasso dello Stato, delle sue istituzioni politiche, sanitarie, scolastiche e, prima di ogni cosa, del cosiddetto "monopolio della violenza legale" già incarnato dal protagonista, un poliziotto che solo gradualmente capirà di avere perso questo ruolo di "tutore 
dell'ordine", o meglio di un ordine che non c'è più, e di essere diventato, suo malgrado, tutore di un nuovo ordine: la sopravvivenza della sua famiglia e del suo gruppo. Come ha scritto Fabrizio Denunzio «Ciò che è stato lungamente sognato da anarchici e rivoluzionari, diventa realtà grazie all'outbreak. Non è più un "fantasma" ad aggirarsi per il mondo e a fare spavento alle potenze capitalistiche - come notavano Marx ed Engels in apertura del Manifesto del partito comunista - ma un'orda di zombi. Il comunismo da spettro è diventato un morto vivente» [Denunzio 2015, 207]. Dallo Stato centralizzato nato in età moderna ed evolutosi tra XIX e XX secolo in Stato-nazione si assiste a una regressione a tanti micro-Stati (quasi delle tribù) rappresentati dalle comunità di sopravvissuti che vengono in contatto tra loro e si scontrano nel corso della serie: la Prigione, Woodbury, Terminus, Alexandria, Hilltop, il Regno, i Salvatori, ecc.

Numerosi sono, infine i riferimenti agli elementi tipici di quel discorso patriottico che una vastissima produzione storiografica [Mosse 2009; Banti 2000] ci ha abituati a vedere come costituivo del processo di fondazione e di costruzione dello Stato-nazione. Tra questi spicca la onnipresente (fin dalle prime scene del primo episodio) bandiera americana che, ad esempio, fa da sfondo al letto del camper (ma appesa al contrario!) nel quale uno dei protagonisti della prima serie, morso da uno zombi, agonizza pre-vedendo la sua futura zombificazione. Il riferimento alla trasformazione della popolazione americana in un'unica orda infinita di zombi è palese [Denunzio 2015, 212].

\section{L'epopea dei pionieri e della Frontiera}

Edwin Jenner: «Perché siete qui? Che volete?».

Rick Grimes: «Una occasione».

TS-19, I/6 


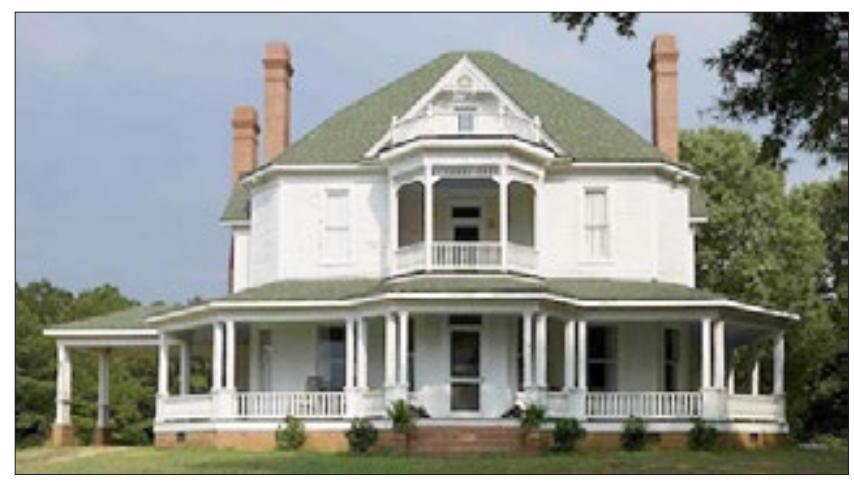

La fattoria di Hershel

Tutta la serie richiama continuamente l'epopea dei primi pionieri americani. Il mondo post-apocalittico, infatti, è diventato un territorio ostile, quasi una terra di conquista, un'esperienza di frontiera come quella vissuta dai primi immigrati europei nel Nuovo Mondo.

Soprattutto quando approdano alla fattoria di Hershel, i sopravvissuti del gruppo di Rick sembrano reincarnare le figure di quei puritani che all'inizio del XVII secolo lasciarono l'Europa per fondare la colonia di Plymouth sulle coste dell'odierno Massachusetts. Come i "padri pellegrini” guidati da William Brewster e imbarcatisi sul Mayflower, Rick e i suoi provano a creare una comunità stabile tra mille pericoli, peripezie e difficoltà. E come nei rapporti tra le prime comunità provenienti dal Vecchio Mondo e le tribù indigene, tra il gruppo capeggiato da Rick e gli altri gruppi di sopravvissuti, fiducia ed ostilità, alleanze e scontri cruenti si alternano nei vari episodi della serie.

I padri pellegrini costruirono delle comunità autosufficienti basandosi sull'etica protestante del duro lavoro. Rick e i suoi cercano di fare lo stesso basandosi però sulla centralità del leader e la difesa del gruppo a ogni costo. La ricerca di risorse fondamentali per la sopravvivenza dei padri fondatori coincide perfettamente con il tentativo dei protagonisti di TWD di procacciarsi quante più provviste, farmaci, munizioni, armi. Una fondamentale differenza consiste però nel fatto che, per quanto possibile, i primi insediamenti dei coloni divennero ben presto forti protetti da palizzate, villaggi e poi vere e proprie città. Le sistemazioni dei sopravvissuti all'apocalisse zombi sono invece sempre provvisorie e 
costringono i protagonisti a una vita nomade che mette a dura prova le loro capacità di adattamento. Le città, da millenni la base della civiltà umana organizzata in società, sono abbandonate e invase dai "morti viventi".

Per i pionieri gli ostacoli e i pericoli venivano dalle tribù indiane ostili e dai banditi. Per i sopravvissuti vengono dalle mandrie dei "vaganti" e dagli altri uomini.

Come i puritani fuggiti dalle persecuzioni del Vecchio Mondo, i superstiti dell'apocalisse zombi sono costretti a ricominciare una nuova vita, a costruirsi una nuova identità e una nuova etica. Essi devono riscrivere le regole del vivere civile in un mondo nuovo (come il Nuovo mondo americano si contrappone al Vechio mondo europeo) cercando di soddisfare innanzitutto i bisogni primari: cibo, acqua, risorse, rifugi. Più che sull'individuo solitario (che pure c'è ed è rappresentato dal leader Rick Grimes), la serie è centrata sul gruppo e sulle sue dinamiche interne. Si tratta di una micro-società, proprio come le prime comunità di coloni nelle quali le differenze esistenti nel Vecchio mondo europeo, di carattere sociale ed economico, erano molto più sbiadite. Allo stesso modo, nel gruppo dei sopravvissuti, tutti i parametri che definivano l'appartenenza, come l'istruzione, la classe sociale o il colore della pelle, perdono significato. Nuove forme di solidarietà prendono forma, cambiando i criteri di inclusione ed esclusione sociale [Grasso, Penati 2016, 199].

I sopravvissuti di TWD capiscono subito che una delle questioni centrali è il potere, a chi affidarlo e come gestirlo. Il mondo post-apocalittico, infatti, sembra a prima vista un ritorno a uno stato di natura privo di istituzioni socio-politiche, quel caos primordiale per il quale l'unico rimedio individuato da Hobbes era appunto il Leviatano, lo Stato centralizzato che avoca a sé l'insieme delle libertà individuali per fornire in cambio benessere e sicurezza collettivi ai suoi cittadini. Nelle piccole comunità di TWD lo Stato è però incarnato da pochi individui (Rick, il Governatore, Negan, il pusillanime e inadeguato Gregor, Deanna 
Monroe ad Alexandria), in quello che sembra un vero e proprio ritorno a una politica di tipo feudale (fino alla rinascita delle antiche monarchie medievali nel Regno, appunto, di Ezekiel, in realtà ex guardiano di uno zoo dal quale ha portato in salvo con sé una tigre che tiene accovacciata a lato del suo "trono").

I pionieri che giunsero sulle coste orientali del continente nordamericano per fondare i primi villaggi in territori sconosciuti e ostili avevano lasciato spesso alle loro spalle (basti pensare ai separatisti puritani della Chiesa anglicana imbarcatisi sul Mayflower nel 1620) persecuzioni, contrasti religiosi e culturali, un'idea del potere inteso come qualcosa di oppressivo. I pionieri dovettero costituire da zero istituzioni, regole, forme di convivenza per sopravvivere e per espandere le proprie comunità. Stipularono quindi tra loro dei patti (covenant) che risalivano all'esperienza dei Padri pellegrini. Approdati a Cape Cod, i passeggeri della Mayflower avevano firmato il Mayflower Compact, una sorta di contratto sociale con il quale si costituivano come corpo politico legittimato a governare e amministrare il loro insediamento coloniale, Plymouth Colony, secondo i principi del bene comune. Un altro importante covenant sarebbe stato quello promosso dieci anni più tardi, negli anni '30 del Seicento, da John Winthrop, il capitano della nave Arbella con la quale arrivò in America una seconda ondata puritana. Un successivo covenant fu quello promosso all'inizio degli anni '60 del XVII secolo dal reverendo Samuel Stoddard, lo Half-Way Covenant. I covenant del Seicento influenzarono il pensiero politico dei padri fondatori degli Stati Uniti e i documenti fondanti della democrazia statunitense (la Dichiarazione di Indipendenza e la Costituzione), con l'idea di un accordo volontario e condizionato, valido soltanto nella misura in cui il governo avrebbe rispettato i diritti inalienabili del popolo.

Questo tipo di "narrazione" è un sottotesto presente in tutte le stagioni della serie. Dopo aver trovato un nuovo e più stabile rifugio (la Prigione) la comunità capeggiata da Rick capisce che deve dotarsi di un Consiglio per prendere decisioni che non possono più pesare su un 
solo uomo, allo stesso modo delle prime comunità di coloni arrivati sulle coste orientali del Nord America. Hanno bisogno di un covenant per affrontare il Governatore di Woodbury, perfetta incarnazione del tiranno da abbattere con la rivoluzione descritto da John Locke, le cui riflessioni sono alla base della dichiarazione d'indipendenza americana.

Oltre a quella dei Padri pellegrini, la vicenda dei protagonisti di TWD ha come precedente storico immediato quello dei pionieri lanciati alla conquista della "frontiera". Essa è metafora di un sogno di libertà e di espansione della nazione americana verso ovest alla ricerca di nuovi territori e di nuove opportunità. Nella prima scena del prologo al primo episodio della serie l'auto di uno sceriffo arriva davanti a quello che, a prima vista, è un semplice incidente stradale. Il vicesceriffo Rick Grimes scende dall'auto. Ha il viso sconvolto. Cerca carburante ma, in mezzo a cadaveri putrefatti e automobili inservibili, trova soltanto una ragazzina zombi alla quale è costretto a sparare in testa. L'episodio termina con lo stesso Grimes che galoppa a cavallo, armato di fucile e di pistola, nella migliore tradizione western e che poi incede lentamente in una città fantasma [Atlanta] priva di segni di vita e immersa nella desolazione più totale. Nel decimo episodio della settima stagione l'immagine dipinta sulla fiancata di un TIR che fa da sfondo alla cazzottata tra Richard e Daryl raffigura un bandito a cavallo che punta le pistole contro una diligenza. Forse una delle immagini più iconiche di tutta una letteratura western scritta e cinematografica. Si tratta soltanto di alcuni dei tanti momenti della serie che fanno esplicito riferimento all'epopea del West. Non è un caso che, come scrivono Aldo Grasso e Cecilia Penati, «fino all'inizio degli anni sessanta, il western rappresenta il principale immaginario di riferimento del racconto seriale televisivo, una rielaborazione del passato mitologico degli Stati Uniti che diventa occasione per dare forma narrativa ad ansie ed inquietudini contemporanee» [Grasso, Penati 2016, 48]. 


\section{Democrazia vs totalitarismo}

Merle Dixon: "Se ti ho dato la caccia erano solo affari, eseguivo gli ordini».

Michonne: «Come la Gestapo».

L’inganno, II/15

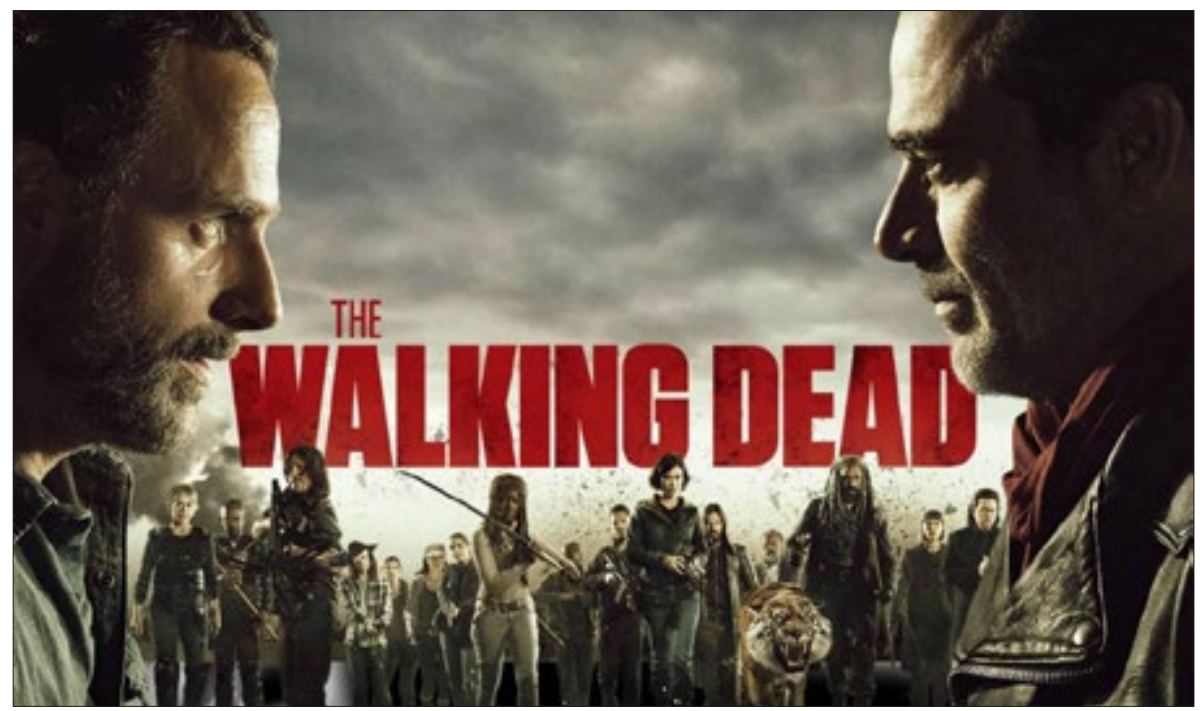

Un manifesto della serie-TV The Walking Dead: Rick e Negan

Il protagonista della serie è il vicesceriffo della contea di King, Rick Grimes. Egli incarna la figura del leader, consapevole che essere al comando di una comunità significa sempre cercare di perseguire il bene comune, anche a discapito dei singoli individui. Grimes non sceglie di diventare il leader. Sono le circostanze e la sua divisa la quale, prima ancora che di stoffa è un "habitus" mentale e psicologico predisposto al comando e alla responsabilità, a determinare il suo ruolo di guida e di autorità surrogata. Non è difficile paragonare questa figura di un leader costretto, quasi suo malgrado, a prendere decisioni, accettarne la responsabilità e portarne il peso, a quella di un paese come gli Stati Uniti. L'America, infatti, dopo la fine della Prima e della Seconda guerra mondiale e soprattutto dopo il crollo dell'Unione sovietica, si è assunta il ruolo di superpotenza globale alla quale tutto il mondo deve guardare 
come esempio e come modello. Fino a sobbarcarsi, apparentemente suo malgrado (vedi la crisi della ex-Jugoslavia o quella della Somalia) compiti ingrati e impopolari di "poliziotto mondiale" e di "paese esportatore" di democrazia.

Nel "secolo americano" gli Stati Uniti si sono sempre proposti come giovane democrazia repubblicana "sostenuta da Dio", investiti del diritto-dovere di combattere le dittature e i totalitarismi. È questa una costante della autorappresentazione dell'America novecentesca (che si è assunta il ruolo di superpotenza alla quale tutto il mondo deve guardare come esempio e modello di democrazia) e quindi in lotta con le dittature per affermare a livello mondiale i valori della Dichiarazione di Indipendenza e della Costituzione americana: da Hitler e Mussolini a Stalin e Mao, da Saddam Hussein fino a Gheddafi.

Lo scontro tra il gruppo di Rick e, di volta in volta, quello di Woodbury, di Negan e dei Salvatori ecc., sembra riproporre questo scontro tipicamente novecentesco.

In effetti, la Woodbury del Governatore, l'antagonista di Rick protagonista della terza stagione, sembra un piccolo stato totalitario di novecentesca memoria. Si tratta di una città fortificata e militarizzata. Il suo leader è dotato di un forte carisma che affascina i suoi concittadini. La volontà di espansione, di conquista e di accaparramento di tutte le risorse disponibili, fino alla decisione di dichiarare una vera e propria guerra alla comunità capeggiata da Rick, ci ricorda il Lebensraum hitleriano e l'aggressiva politica estera e militare della Germania nazista. Come il Governatore, Hitler voleva creare una "Germania prospera e felice" ma a discapito della libertà e della ricchezza di altri Stati e di altri popoli. I duelli all'ultimo sangue tra zombi affamati e le feste che il Governatore organizza ci richiamano quel panem et circenses che i dittatori (e prima ancora gli imperatori) hanno sempre considerato alimento indispensabile del consenso delle masse verso i propri governi. Il Governatore, come ogni dittatore interessato più al proprio potere che al benessere della comunità, è pronto a eliminare i suoi stessi uomini se 
questi lo deludono. Considera gli abitanti di Woodbury come qualcosa di suo; con Rick è disposto a trattare solo una resa incondizionata; finge un appeasement ma in realtà prepara un attacco massiccio e definitivo contro la Prigione. Rick invece è pronto a fare un passo indietro per il bene della sua comunità.

Il Governatore è uno spietato assassino e allo stesso tempo un bugiardo che mente alla sua comunità, nascondendo la sua vera natura, collezionando teste di zombi in un acquario, bevendo alcool spesso e volentieri. Si presenta come un profeta, l'uomo "della provvidenza" capace di conquistare il mondo e di ricostruire una nuova umanità. Capisce che la gente ha bisogno di sicurezza e di certezze e sa fare uso della propaganda per propalare le sue bugie. Basa il suo potere sulla mistificazione e sulla paura e può contare sulla fedeltà dei suoi perché sfrutta il loro bisogno di sicurezza. Woodbury è una cittadina sicura, ben recintata e sorvegliata da guardie armate. All'interno, apparentemente, i suoi cittadini hanno tutto ciò di cui hanno bisogno e vivono quasi una vita normale. Non sanno o fingono di non sapere (come i tedeschi della Germania nazista?) che il loro "benessere" è il frutto delle razzie e delle rapine a danno di altre comunità di sopravvissuti (come dice il Governatore nel settimo episodio della quarta stagione: «Le persone credono a quello a cui vogliono credere. Tutti amano gli eroi»). Uniformarsi è più facile. La comunità di Woodbury è più numerosa, meglio armata $\mathrm{e}$ meglio organizzata di quella di Rick. Le due comunità, proprio come le democrazie occidentali e i totalitarismi nazifascisti alla metà del XX secolo, sono destinate allo scontro. Anche in questo caso sono due visioni diverse, e per certi versi opposte, a confliggere.

La donna che nel terzo episodio della terza stagione magnifica l'azione del Governatore, e che risponde all'obiezione di Andrea sugli zombi appesi agli alberi in modo macabro: «Non li giustifico ma si prendono cura di noi», fa venire in mente la risposta che una tedesca diede allo storico Allen: «Le fila della NSDAP erano piene di giovani. Quella gente seria che ne entrava a far parte lo faceva perché voleva la giustizia sociale, o perché s'opponeva alla disoccupazione. I nazisti suscitavano una sensazione di instancabile energia», Allen 2005, 26. 
Il Governatore promette ad Andrea che non attaccherà la prigione dove si sono rifugiati Rick e i suoi e arriva a parlamentare con lui. In realtà prepara un attacco massiccio contro la prigione per il quale recluta ogni cittadino di Woodbury in grado di imbracciare un'arma, compresi ragazzi e ragazze dai tredici anni in su. Esattamente come farà un Hitler ormai disperato asserragliato nel bunker e che rifita categoricamente di arrendersi, arruolando migliaia di adolescenti nella battaglia finale contro i soldati dell'Armata rossa. La stessa cosa farà il Governatore nell'ottavo episodio della quarta stagione, quando illuderà i suoi ostaggi, Michonne e Hershel, facendo loro credere di voler conquistare la Prigione in modo pacifico. In realtà il Governatore vuole scatenare una vera e propria guerra mosso da ambizione, rabbia, sete di vendetta e mania di controllo proprio come l'Hitler che voleva dare corso alle sue mire espansionistiche, già messe per iscritto sul Mein Kampf. E come il dittatore, il Governatore riesce a sfuggire ad alcuni attentanti contro la sua persona (da parte di Michonne e di Andrea).

D'altra parte Rick accetta di entrare in guerra quando si rende conto che solo in questo modo può salvaguardare la sua comunità. Nonostante la frase con la quale ha chiuso l'episodio della seconda stagione ("Questa non è più una democrazia») Rick continua a credere in alcuni dei valori fondanti dei sistemi democratici. Quando il Governatore gli propone uno scambio (Michonne in cambio della tregua), Rick tentenna. Il Rick padre vorrebbe salvare la sua famiglia. Ma come leader ha già fatto la sua scelta. Nel quindicesimo episodio confessa ai suoi di essere stato sul punto di consegnare Michonne ma ammette di avere sbagliato e capisce che non può decidere da solo: "Volevo consegnare Michonne per la nostra sicurezza. Ma non spetta a me decidere tutto. Siamo noi il bene più grande. Come vivete, come morite, non spetta a me decidere. Non sono il vostro Governatore. Votiamo. Possiamo restare e combattere o possiamo andarcene». Quindi mette ai voti la decisione [Poli 2015, 178]. Rick ridiventa il capo democratico opposto al Governatore-dittatore. Tutto il suo discorso è caratterizzato dalla ri- 
petizione della parola "Noi", quella con la quale inizia la Costituzione americana.

Il Governatore, incarnando gli istinti più bassi e più mostruosi dell'essere umano, ha deciso di "sterminare" il nemico per appropriarsi dello "spazio vitale" (in questo caso la Prigione). Il nemico è ormai chiunque (anche tra i suoi concittadini) non sia d'accordo con lui e con la sua visione del mondo.

L'ultimo passo è la disumanizzazione dell'avversario. Hitler degrada gli ebrei e gli slavi alla categoria di "sottouomini" (Untermensch). Per incitare i suoi allo scontro finale contro la comunità della Prigione il Governatore non esita a paragonare Rick e i suoi uomini agli zombi. Il Governatore finirà per uccidere i suoi stessi uomini stanchi di combattere e il suo potere crollerà come un castello di carte perché basato sulle menzogne, sulla follia e sulla paura. E come Hitler nel bunker che vuole trascinare nel baratro della sua rovina l'intera Germania pur di non arrendersi, dopo la sua sconfitta, il Governatore decide di distruggere Woodbury e di farla invadere dagli zombi.

\section{La wilderness, la controcultura, il femminismo}

Eastmann: «Ho iniziato a convincermi che tutte le vite sono preziose, le persone sono le cose più importanti».

Qui non è qui, VI/4

Daryl Dixon (porgendo una rosa a Carol): «È una rosa cherokee. La storia dice che quando i soldati americani stavano deportando gli indiani dalle loro terre, durante il "Sentiero delle Lacrime", le madri cherokee erano addolorate e piangevano così tanto perché stavano perdendo i loro piccoli lungo il cammino per assideramento, malattie e fame. Molti di loro scomparirono e basta. Così gli anziani innalzarono una preghiera chiedendo un segno per risollevare lo spirito delle madri, per dar loro la forza e la speranza. Il giorno dopo questa rosa cominciò a 
crescere nei posti in cui caddero le lacrime di quelle madri. Non sono così stupido da pensare che un qualsiasi fiore possa sbocciare per mio fratello. Ma credo che questo sia sbocciato per tua figlia».

La rosa Cherokee, II/4

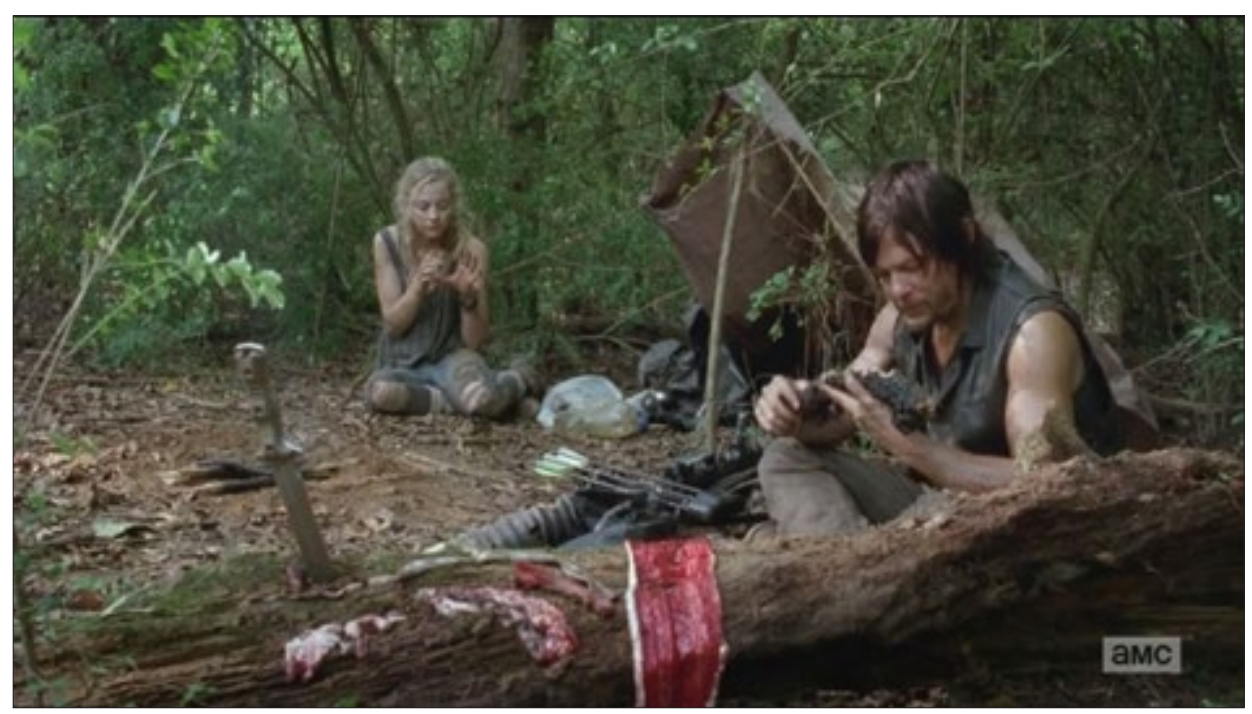

Un'immagine della serie-TV The Walking Dead

Grazie ai personaggi di Morgan e di Carol, il mito della wilderness si associa ai concetti di fuga regressiva, rifugio, meditazione e rigenerazione. Il primo decide di seguire la strada della nonviolenza indicatagli da Eastman e di vivere appartato e solitario proprio per poter assecondare senza "interferenze" esterne le proprie inclinazioni pacifiste. La seconda decide di staccarsi dal gruppo, consapevole che il proprio anticonformismo potrebbe mettere in pericolo i suoi amici (che pure ha salvato in numerose occasioni grazie alle sua abilità e scaltrezza). Essa, più di tanti altri, sembra portatrice di quella self-reliance che era alla base della riflessione dei "trascendentalisti".

Ma più di Morgan e di Carol è Daryl Dixon il personaggio che incarna la tensione verso la wilderness, non solo come rifuto della civilizzazione capitalistico-borghese, ma anche come aspirazione verso un mondo 
più autentico e genuino, contro gli artefatti della civiltà, con le sue ipocrisie e le sue doppiezze. La sua storia personale e familiare lo ha abituato a vivere e a sopravvivere nei boschi e nella natura selvaggia e ostile, imparando molte cose da esperienze dure ed estreme. Egli ricorda per certi aspetti la cultura e la capacità dei nativi americani di vivere a contatto con la natura, sfruttandone le potenzialità ed evitandone i pericoli. La sua abilità con l'arco e le frecce ne sono una conferma. Non è un caso che sarà proprio lui a raccontare a Carol una leggenda cherokee quando cercherà di darle una speranza sulla sorte della figlia scomparsa. A incarnare una cultura di adesione alla natura nutrita dal rispetto della vita e dalla pratica della non-violenza è invece il Morgan che ritorna nella sesta stagione come un personaggio molto diverso rispetto a quello di prima. Nel secondo episodio della sesta stagione, infatti, vediamo un Morgan restio ad uccidere gli aggressori e armato solo di un bastone con il quale pratica l'aikidō. Nel quarto episodio conosciamo i motivi della sua conversione. Un giorno ha trovato una casa nel bosco abitata da un uomo che però riesce a sopraffarlo. Questi tuttavia lo risparmia e lo prende prigioniero, cercando subito dopo di stringere amicizia con lui. L'uomo, di nome Eastman, gli racconta di essere uno psichiatra forense. Dopo vari tentativi, riesce a guarire Morgan dalla sua aggressività grazie all'insegnamento dell'aikidō. Eastman gli racconta che sua moglie e i suoi figli sono stati uccisi da uno dei suoi pazienti. Dopo essersi vendicato uccidendo a sua volta l'assassino, Eastman comprende l'inutilità della vendetta e capisce che ogni vita è preziosa e non bisogna uccidere nessuno. Ma a causa di una distrazione di Morgan, Eastman viene morso da un "vagante". Dopo aver seppellito l'amico, Morgan

La leggenda narra della fioritura di rose lungo il sentiero percorso dalle donne cherokee deportate insieme ai propri figli verso riserve inospitali. La rosa simboleggia la forza che la natura forniva alle donne che rischiavano di perdere i propri bambini per le loro pessime condizioni di vita. I cinque petali della rosa simboleggiavano le cinque tribù indiane deportate: Cherokee, Cickasaw, Choctaw, Creek e Seminole. I petali erano bianchi come le lacrime versate dalle donne, il pistillo era giallo come l'oro per il quale i bianchi avevano scacciato gli indiani dalle loro terre. 
decide di vivere rispettando gli insegnamenti di Eastman e porta con sé la copia de L'arte della pace di Morihei Ueshiba, il fondatore dell'aikidō. In questo modo però metterà in pericolo la comunità di Rick, quando lascerà libero, anziché ucciderlo, uno degli aggressori di Alexandria (i Lupi).

Altra tematica attuale affrontata in molti episodi è quella del femminismo e del rapporto tra i sessi. Nell'accampamento provvisorio sorto non molto lontano da Atlanta, dove Rick trova un gruppo di superstiti (tra i quali la moglie, il figlio e il collega Shane), i ruoli sono ben definiti. I maschi pensano alla protezione e alla difesa della comunità dagli zombi. Le donne sono addette alle mansioni "domestiche": cucinare, accudire i bambini ecc. La divisione sessista dei ruoli sembra fare un salto nel passato, almeno fino agli anni '50. Basti pensare che Shane mette con molta più facilità un'arma nelle mani del figlio di Rick, che è ancora un bambino, piuttosto che in quelle di Andrea, che pure è una ex avvocata e certo non abituata a una vita da casalinga. Ma già nel corso della prima stagione, con il diffondersi della "epidemia zombi", anche le donne devono cominciare a emanciparsi, addestrandosi all'uso delle armi e rendendosi autonome rispetto ai tradizionali ruoli di genere. I due personaggi più importanti, da questo punto di vista, sono Carol e Andrea. La prima è la vittima di un marito violento e maschilista che avrà una vera e propria evoluzione "femminista", fino a diventare un punto di riferimento nel futuro nuovo "assetto militare" del gruppo capeggiato da Rick. La seconda, provata dalla morte della sorella e disgustata dalla paura che l'ha paralizzata in alcune occasioni, decide di diventare padrona della sua vita, imparando a maneggiare una pistola. Altre "donne forti" sono Maggie, figlia di Hershel e Michonne, la donna guerriera armata di katana che diventerà uno dei personaggi più importanti per la sopravvivenza del gruppo e compagna del leader. Il ruolo centrale acquisito dalle donne è uno dei punti di forza delle nuove narrazioni presenti in molte serie tv di inizio XXI secolo, an- 
cora più rilevante nella visione statunitense rispetto a quella britannica [Moroni 2016, 139]. Anche in TWD la figura della donna diventa una risorsa simbolica del nuovo mondo. La figlia di Lori (non sappiamo se di Rick o di Shane) è, appunto, una bambina. A essa è affidato il messaggio di speranza che anche in un mondo post-apocalittico l'umanità sopravvive e si riproduce.

La comunità nella quale si imbatte Tara nel sesto episodio della settima stagione è formata da sole donne. La leader di questa comunità rivela a Tara che tutte le persone di sesso maschile di oltre 10 anni sono state uccise dai Salvatori. Si tratta di un'estremizzazione delle teorie femministe sul "potere della donna" che, in questo caso, si basa sull'eliminazione dell'elemento maschile. Con esso però finirebbe la possibilità della riproduzione, a conferma della mancanza di speranza per il futuro del genere umano in un mondo post-apocalittico.

\section{Il passato, la storia e la memoria}

Edwin Jenner: «Pensiamo sempre che ci sarà ancora tempo. Poi però, il tempo finisce».

TS-19, I/6

Nel quarto episodio della prima stagione Morales nota l'orologio al polso di Dale e gli dice:«Devo chiedertelo, mi sta facendo impazzire. L'orologio. Ti vedo ogni giorno alla stessa ora caricare quel coso come il prete al villaggio che dice messa». E Dale risponde: «È il tempo, è importante tenerne il conto, quanto meno dei giorni. Sapete, mi piace quello che un padre disse al figlio quando gli diede un orologio che era stato tramandato da generazioni». E subito dopo cita L'urlo e il furore di William Faulkner: «Quentin, eccoti il mausoleo di ogni speranza e desiderio; è molto probabile, purtroppo, che te ne serva anche tu per ottenere il reducto absurdum di ogni umana esperienza, che non farà per i tuoi bisogni individuali piú di quanto fece per i suoi o per quelli di 


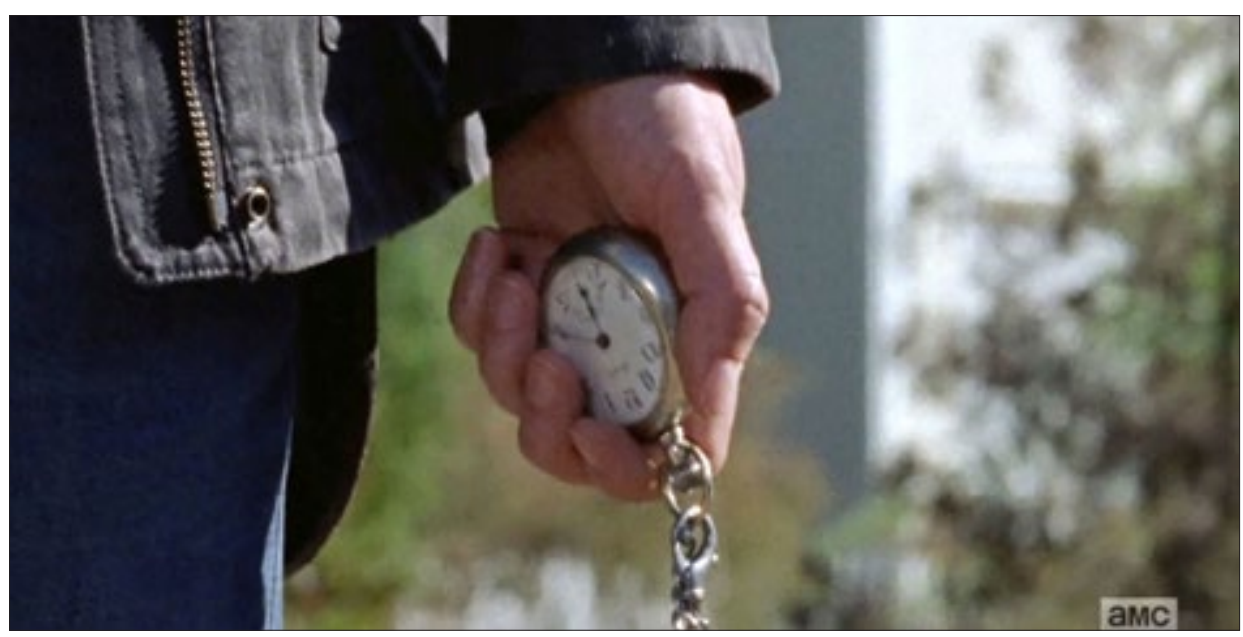

Uno dei tanti orologi "protagonisti" in The Walking Dead

suo padre. Non te lo do perché tu possa ricordarti del tempo, ma perché ogni tanto tu possa dimenticarlo per un attimo e non sprecare tutto il tuo fiato nel tentativo di vincerlo» [Faulkner 1997, 67].

Comprensibilmente, i riferimenti al passato, alla memoria e alla storia non possono essere frequenti in una serie tv che parla di un futuro apocalittico e di un nuovo mondo del quale si sottolineano continuamente le differenze con il vecchio. Tuttavia se ne possono cogliere alcuni particolarmente indicativi.

Nel dodicesimo episodio della terza stagione, il figlio del protagonista, Carl, che vediamo crescere nel corso delle varie stagioni, decide di andare a prendere una foto nella casa dove viveva con suo padre e sua madre prima dell'apocalisse. Carl rischia la vita per recuperare una foto di sua madre che è morta dopo aver dato alla luce una bambina. Nel nuovo mondo la memoria deve affidarsi ai ricordi perché i video, i telefoni, le tv non esistono più. Ma i bambini come Judith, la nuova sorella di Carl, sono troppo piccoli per ricordare perché sono nati nella nuova era. Essi dunque hanno bisogno di supporti, fotografici e documentari, per ricordare. Ecco perché Carl prende la foto: per far vedere a Judith quanto era bella la sua mamma e come erano felici i suoi genitori. Perché prima o poi bisogna fare i conti con il passato [Poli 2015, 168]. 
Le esperienze vissute dai protagonisti di TWD dimostrano non solo che il "vecchio mondo" con le sue regole etiche, sociali e civili non esiste più, ma anche che nel "nuovo mondo" non servono più i vecchi valori dell'esperienza, del buonsenso, della saggezza. Questi sono incarnati da due personaggi come Dale e Hershel, gli anziani del gruppo, i quali, infatti, finiscono per soccombere. Il Nuovo mondo è un mondo "giovane" nel quale sono pochi i cinquanta-sessantenni. Nel mondo post-apocalittico non ci sono più prove o testimoni. Non ci sono più documenti. La storia sembra una disciplina inutile e impossibile. Ma prima o poi i sopravvissuti dovranno fermarsi in modo più stabile e, se riusciranno a sconfiggere gli zombi e a trovare un antidoto per l'epidemia, dovranno sforzarsi di ricostruire il proprio passato per lasciare ai Carl e alle Judith una memoria di quello che era il mondo prima dell'apocalisse e quello della lunga "traversata nel deserto". E a quel punto avranno di nuovo bisogno di storici i quali utilizzeranno qualsiasi tipo di documento, nel senso braudeliano del termine, per ricominciare a scrivere la storia.

Nel tredicesimo episodio della terza stagione, Milton Mamet, il consigliere di Negan, scrive qualcosa su un taccuino e poi dice: «La chiamerei battaglia e l'ho fatto. L'ho raccontata. Qualcuno deve descrivere quello che abbiamo passato. Sarà parte della nostra storia». Nell'ottavo episodio della sesta stagione Eugene, nascosto dentro un garage per sfuggire agli zombi, legge un libro intitolato World History.

Un momento fortemente simbolico di un passato che ritorna è poi rappresentato nell'undicesimo episodio della seconda stagione dal passaggio di testimone tra Hershel e Glenn. Quando il primo viene a sapere della relazione tra Glenn e la figlia Maggie, Hershel accoglie il ragazzo in seno alla famiglia affidandogli l'orologio da tasca di suo nonno. Nel quindicesimo episodio della terza stagione Glenn e Hershel parlano dell'orologio e il primo dice: "Ora so cosa significa». Glenn ha capito che l'orologio è il simbolo della continuità, della memoria e del passato che li accompagna nel presente e nel futuro. 
L'orologio, simbolo del tempo che passa e del passato (quindi della memoria e della storia) ricompare nelle mani di Maggie nell'ultima inquadratura della settima stagione e al polso di Rick nel primo episodio dell'ottava.

\section{Bibliografia}

Allen W. S. 2005, Come si diventa nazisti, Torino: Einaudi.

Banti A.M. 2011, La nazione del Risorgimento. Parentela, sanità e onore alle origini dell'Italia unita, Torino: Einaudi.

Bertella Farnetti P., Bertucelli L., Botti A. (eds.) 2017, Public History. Discussioni e pratiche, Milano-Udine: Mimesis.

Cipolloni M. 2017, Monkey \& Business. Storia naturale e storia umana sul grande schermo, in Bertella Farnetti P., Bertucelli L., Botti A. (eds.) 2017, Public History. Discussioni e pratiche, Milano-Udine: Mimesis, 243-268.

Denunzio F. 2015, L'outbreak come collasso dello spazio e fine dello Stato, in Frezza G. (ed.), 2015, Endoapocalisse. The Walking Dead, l'immaginario digitale, il post-umano, Salerno: Areablu edizioni, 199-213.

Grasso A., Penati C. (eds.) 2016, La nuova fabbrica dei sogni. Miti e riti delle serie tv americane, Milano: Il Saggiatore.

Faulkner W. 1997, L'urlo e il furore, Torino: Einaudi.

Moïsi D. 2016, La géopolitique des séries, ou le triomphe de la peur, Paris: Stock.

Moroni S. 2016, "Mainstream": il XX secolo narrato attraverso i period dramas angloamericani (2010-2014), «Passato e presente», 98: 133-150.

Mosse G. L. 2009, La nazionalizzazione delle masse. Simbolismo politico e movimenti di massa in Germania (1812-1933), Bologna: il Mulino.

Noiret S. 2009, "Public History" e "Storia Pubblica" nella rete, "Ricerche storiche", XXXIX (2-3): 275-327.

Noiret S. 2017, Introduzione: per la Public History internazionale, una disciplina globale, in Bertella Farnetti P., Bertucelli L., Botti A. (eds.) 2017, Public History. Discussioni e pratiche, Milano-Udine: Mimesis, 9-34.

Poidomani G. 2016, I Simpson e la storia. Viaggio nel tempo a bordo di un divano, Milano: Sironi.

Poli C. 2015, C’è un solo Leader. Anatomia della serie tv "The Walking Dead”, Saldapress: Reggio Emilia. 
Ridolfi M. 2017, Verso la Public History. Fare e raccontare storia nel tempo presente, Pisa: Pacini Editore.

Testi A. 1996, Il passato in pubblico: un dibattito sull'insegnamento della storia nazionale negli Stati Uniti, «Storica», 6: 7-53.

Vezzosi E. (ed.) 2009, I festival di storia e il loro pubblico, "Contemporanea», 4: 717-719. 